\title{
Estudos clínicos sobre úlcera por pressão
}

\author{
Clinical studies on pressure ulcer
}

Estudios clínicos sobre úlcera por presión

\section{Thaís Sanglard de Souza', Otília Beatriz Maciel', Marineli Joaquim Méier', Mitzy Tannia Reinchembach Danski', Maria Ribeiro Lacerda' \\ 'Universidade Federal do Paraná. Programa de Pós-graduação em Enfermagem. Grupo de Estudos Multiprofissional em Saúde do Adulto. Curitiba, PR}

Submissão: 09/07/2009

Aprovação: 17/05/2010

\section{RESUMO}

O estudo teve como objetivo sintetizar os estudos clínicos sobre úlcera por pressão (UP). Elaborou-se uma revisão integrativa através de busca de artigos nas bases de dados MEDLINE, Portal de Evidências, LILACS, e o periódico Joanna Briggs Institute (JBI), referência na área de ensaios clínicos em enfermagem, no período de 2004-2009. Selecionou-se 14 artigos e a análise destes permitiu a identificação de euatro categorias temáticas envolvendo a caracterização dos estudos, da amostra, das intervenções utilizadas, bem como dos resultados e conclusões alcançadas. Os resultados indicaram essencialmente o enfoque de estudos direcionados a temática da prevenção e a ausência desse tipo de estudo em nosso país. Reconhecer as limitações envolvidas nesse aspecto e buscar superá-las proporcionará avanços e impulsionará o desenvolvimento de pesquisas com fortes evidências clínicas Que subsidiem a prática profissional da enfermagem.

Descritores: Pesquisa clínica; Úlcera por pressão; Enfermagem.

\section{ABSTRACT}

The present study aimed at providing a synthesis of clinical studies on pressure ulcer (UP). An integrative review was made by searching the database of MEDLINE, Portal de Evidências, and LILACS for articles, and the Joanna Briggs Institute (JBI) periodical, a reference in clinical trials in Nursing, from 2004-2009. 14 articles were selected and their analysis made it possible to identify four areas of interest involving the characterization of the studies, the sample, the interventions used, as well as the results and conclusions obtained. We found that the studies reviewed have focused on prevention and that there are few studies of this kind in our country. It is important to recognize these limitations and try to overcome them in order to provide advancements and enhance the development of research based on strong clinical evidence to support the professional nursing practice.

Key words: Clinical research; Pressure ulcer; Nursing.

\section{RESUMEN}

El estudio tuvo como objetivo sintetizar los estudios clínicos sobre úlcera por presión (UP). Fue elaborada una revisión integradora a través de búsqueda de artículos en las bases de datos MEDLINE, Portal de Evidencias, LILACS, y el periódico Joanna Briggs Institute (JBI), referencia en el área de ensayos clínicos en Enfermería, en el periodo de 2004-2009. Se seleccionaron 14 artículos y el análisis de los mismos permitió la identificación de cuatro categorías temáticas envolviendo la caracterización de los estudios, de la muestra, de las intervenciones utilizadas, bien como de los resultados y conclusiones alcanzados. Los resultados indicaron esencialmente el enfoque de estudios direccionados a la temática de la prevención y la ausencia de este tipo de estudio en nuestro país. Reconocer las limitaciones envueltas en este aspecto y buscar superarlas, proporcionará avanzos e impulsará el desarrollo de investigaciones con fuertes evidencias clínicas Que subsidien la práctica profesional de la enfermería.

Descriptores: Investigación clínica; Úlcera por presión; Enfermería. 


\section{INTRODUÇÃO}

O enfermeiro, membro da equipe multiprofissional de saúde e líder da equipe de enfermagem é responsável pelo gerenciamento do cuidado. Gerenciamento este, Que destaca a tomada de decisão, e Que propicia a escolha da melhor prática de cuidar a ser empregada ao paciente hospitalizado. Entretanto, para uma prática com Qualidade, faz-se necessário Que as ações de cuidado estejam cientificamente sustentadas na melhor evidência clínica, para a otimização dos recursos humanos disponíveis e a redução de custos à instituição.

Dentre os tratamentos Que geram altos custos as instituições de saúde evidenciam-se os destinados às úlceras por pressão (UP) ${ }^{(1)}$. Também denominadas como úlceras de decúbito, são definidas como uma área de dano localizado na pele e estruturas subjacentes devido à pressão ou fricção e/ou combinação destes ${ }^{(2)}$.

As UP influenciam drasticamente o período de hospitalização com repercussões diretas no desconforto e dor ocasionados aos pacientes acometidos por estas lesões. Estudos apontam elevados índices de prevalência e incidência de UP em pacientes hospitalizados ${ }^{(3-4)}$. Devido a gravidade do problema Que as UP podem ocasionar as mesmas foram incluídas pela Agency for Health Care Polycy and Research (ACPHR) como um dos indicadores de Qualidade da assistência a saúde.

Para o eficaz gerenciamento relacionado tanto as práticas curativas Quanto preventivas das UP, é necessário Que o enfermeiro mantenha-se constantemente vinculado as bases científicas do cuidado, a pesQuisa, consciente da sua relevância para a prática.

No atual contexto em Que a profissão de enfermagem se insere as pesquisas clínicas subsidiam a Prática Baseada em Evidências (PBE). Esta envolve a definição de um problema, a busca e a avaliação crítica das evidências disponíveis, a sua implementação na prática e a ponderação ou julgamento dos resultados obtidos, de modo a encorajar uma assistência à saúde fundamentada em conhecimento científico, com resultados de Qualidade e custo efetivo(5). $^{(5)}$.

No movimento de PBE há necessidade de produção de métodos de revisão de literatura, os Quais permitem a procura, a avaliação crítica e a síntese das evidências disponíveis acerca do tema investigado. Dentre estes temas destaca-se o método da revisão integrativa ${ }^{(6)}$. Este método permite Que o leitor reconheça os profissionais Que investigam sobre determinado assunto, classificar os achados científicos, além de descrever o conhecimento no seu estado atual, promovendo o impacto sobre a prática clínica.

No intuito de contribuir para o gerenciamento das ações envolvidas no manejo das UP e conhecer o Que se tem desenvolvido na prática clínica de enfermagem em âmbito nacional e internacional o presente estudo objetivou analisar os estudos clínicos sobre UP na enfermagem.

\section{METODOLOGIA}

Utilizou-se no estudo o método de revisão integrativa constituído em seis etapas: seleção das hipóteses ou Questões para a revisão; estabelecimento de critérios de seleção de amostra; categorização dos estudos e síntese do conhecimento produzido; análise de dados e resultados; e interpretação dos resultados(7).
A primeira etapa diz respeito ao estabelecimento do problema de revisão, é uma etapa de formulação de hipóteses ou Questões para a investigação. A construção da Questão da pesquisa é relacionada a um raciocínio teórico e tem por base definições já apreendidas pelo pesQuisador ${ }^{(7)}$. A presente revisão tem como Questão norteadora: "Quais as melhores práticas clínicas destinadas ao manejo das UP na enfermagem"?

A segunda etapa refere-se a seleção da amostra, é a etapa de critérios de inclusão e exclusão de artigos, em Que ocorrerá a seleção das pesquisas Que serão revisadas ${ }^{(7)}$. Foi realizada uma busca nas bases de dados LILACS, Que reúne as publicações científicas da área da Saúde da América Latina e do Caribe; MEDLINE, areuivo digital produzido pela National Library of Medicine (USA) na área das Biociências; Portal de Evidências, o Qual oferece acesso integrado a fontes de informação em saúde de melhor nível de evidência científica; e o periódico Joanna Briggs Institute (JBI), referência na área de ensaios clínicos em Enfermagem, sendo que os descritores selecionados para busca dos artigos foram: pesquisa clínica (clinical tria), úlcera por pressão (pressure ulcer) e enfermagem (nursing). Foram utilizadas as palavras - chave consideradas como descritores no DECs e MESH.

Os critérios de inclusão no presente estudo foram: artigos indexados nas bases de dados mencionadas e publicados na íntegra em periódicos nacionais e internacionais; compreendidos entre o período de 2004 a 2009 e disponíveis nos idiomas português, inglês e espanhol.

Para a seleção das publicações, foi lido cada título e resumo de modo a confirmar se eles contemplavam a pergunta norteadora desta investigação e se atendiam aos critérios de inclusão e exclusão estabelecidos.

Ao efetuar-se a busca nas bases de dados LILACS e MEDLINE não encontrou-se nenhum artigo com os descritores e limites anteriormente estabelecidos.

Das 12 referências selecionadas na base Portal de Evidências duas foram excluídos da análise, visto Que uma não estava relacionado à temática e uma disponível apenas no idioma alemão, não contemplando o critério de inclusão pertinente ao idioma. Nesta base obteve-se 10 artigos.

Foram encontradas 07 referências na base de dados PUBMED, entretanto, 02 artigos já haviam sido localizados na base de dados Portal de Evidências e, portanto, foram excluídos e 03 artigos não contemplavam o tema de estudo. Ao final foram totalizados 02 artigos.

O periódico JBI apresentou três referências, todavia, uma não retratava a temática e foi excluída, restando dois artigos para análise.

Após percorrer-se a trajetória descrita anteriormente, selecionouse ao todo 14 artigos Que contemplavam a pergunta norteadora do nosso trabalho e Que também atendiam aos critérios previamente estabelecidos.

A terceira etapa contempla a categorização dos estudos, de modo a realizar uma extração das informações Que permita organizar e sumarizar os achados dos estudos e confeccionar a formação de bancos de dados ${ }^{(7)}$. Para a extração dos dados dos artigos incluídos na revisão integrativa, foi elaborado e adotado um instrumento contemplando os itens: identificação do artigo original, características metodológicas do estudo, avaliação do rigor metodológico, intervenções estudadas e resultados encontrados. 
A síntese dos dados extraídos dos artigos foi apresentada de forma descritiva em tabelas, reunindo o conhecimento produzido sobre o tema investigado nesta revisão integrativa. As Quatro categorias temáticas foram elaboradas a partir dos temas abordados nos artigos analisados.

A Quinta e sexta etapas da revisão integrativa é a análise e discussão de resultados e interpretação dos resultados devem incluir informações suficientes para Que o leitor possa examinar criticamente as evidências levantadas e suas implicações para a prática da pesquisa $^{(7)}$.

\section{RESULTADOS}

Ao analisar os 14 artigos selecionados foi constatado Que em relação à autoria $5(35,7 \%)$ eram de enfermeiros; 1 ( $7,1 \%)$ de enfermeira e médico; $6(42,8 \%)$ de enfermeiros, médicos e profissionais de outras áreas como estatísticos, epidemiologistas e engenheiros; e em dois artigos (14,2\%) não foi possível identificar a formação dos autores.

Os temas abordados foram: materiais usados na prevenção de UP (05), tratamentos de UP (03), escalas de avaliação de risco para o desenvolvimento de UP (02), comparações entre a concordância de profissionais de saúde acerca das práticas clínicas relacionadas às $\mathrm{UP}^{(4)}$.

Em relação à instituição em Que os estudos foram desenvolvidos, obteve-se oito hospitais, um multicêntrico, um laboratório, um domicílio, um estudo não possuía identificação e duas revisões sistemáticas da literatura obtiveram suas informações nas bases de dados eletrônicas.

Quanto ao tipo de revista científica II foram publicações de enfermagem, um periódico médico, dois de outras áreas da saúde.

Ao analisar os delineamentos de pesquisa dos artigos incluídos, constatou-se Que 7 (50\%) tratam-se de ensaios clínicos randomizado, $2(14,2 \%)$ pesquisa com delineamento Quaseexperimental, ou seja, ensaio clínico sem randomização, $3(21,4 \%)$ estudos com delineamento não experimental, sendo todos descritivos e duas revisões sistemáticas da literatura (14,2\%).

Em relação as forças de evidência observou-se dois artigos com nível de evidência I, sete artigos com nível de evidência II e dois artigos com nível de evidência III, sendo três artigos com nível de evidência $V^{(8)}$.

Os Quadros 1 a 4 (no APÊNDICE) apresentam a síntese dos artigos incluídos na revisão integrativa em Quatro categorias temáticas, respectivamente intituladas: materiais utilizados na prevenção de UP; diferentes tratamentos usados em UP; escalas de avaliação de risco para o desenvolvimento de UP e, comparações entre a concordância de profissionais de saúde acerca das práticas clínicas relacionadas às UP.

As Quatro categorias temáticas nos permitiram inferir Que a preocupação acerca da temática de úlceras por pressão é de ampla magnitude e envolve tanto recursos materiais Quanto humanos em seus aspectos de prevenção, tratamento e aprimoramento de tecnologias de cuidado de enfermagem.

\section{DISCUSSÃO}

Os estudos realizados em países europeus ${ }^{(10-13,17-22)}$ apareceram como líderes das publicações de pesquisas clínicas sobre UP seguidos pela Austrália ${ }^{(12,15-16)}$ e Ásia ${ }^{(9)}$, enQuanto no Brasil, nenhuma pesquisa foi publicada no período de 2004 a 2009. É urgente o investimento na pesquisa nessa área, para compreensão da importância da prevenção das UP, seu impacto econômico às instituições de saúde e na efetividade do cuidado prestado.

A revisão possibilitou identificar Que dentre os 14 artigos, em seis $^{(9-10,12-13,15,18)}$ o enfoque foi a temática da prevenção. Assim, algumas observações são pertinentes: a primeira diz respeito a incipiência de estudos clínicos Que abordem essa temática, e a segunda a necessidade destes estudos direcionados para a prevenção UP além do seu tratamento.

No âmbito do tratamento das UP, a realização de curativos com produtos adequados é essencial para a evolução da cicatrização da ferida bem como para a redução de custos para as instituições de saúde Que por fatores diversos não obtiveram sucesso com a prevenção das UP. Destaca-se nesse ínterim a relevância de estudos Que avaliem a efetividade dos tratamentos propostos, a exemplo das revisões sistemáticas abordadas em dois dos trêsartigos dessa revisão integrativa.

Evidencia-se o uso da escala de Braden como instrumento de avaliação de risco para o aparecimento de UP em dois artigos ${ }^{(18,12)}$. A escala de Braden tem os melhores valores preditivos reportados constituindo-se em um ótimo referencial agregado ao julgamento clínico das enfermeiras ${ }^{(18)}$.

Durante o processo de inspeção da pele em sítios considerados críticos para o desenvolvimento de UP, a escala de classificação adotada para o estagiamento dos níveis, mencionada em seis $\operatorname{artigos}^{(10,13,17-19,21)}$ incluídos nesta revisão integrativa adotaram o sistema de classificação adotado pela EPUAP (European Pressure Ulcer Advisory Panel) com graus de estagiamento Que variam de I a IV.

Outro aspecto significativo foi referente ao Quadro 3, a identificação do Grau I da UP, o Qual é definido pela EPUAP como eritema não branqueável. Tal reação não embranQuece após a remoção da pressão; descoloração, calor, edema, endurecimento ou dureza sendo utilizada como indicador de alerta para desenvolvimento de UP em graus mais avançados especialmente em indivíduos com pele mais escura.

O Quadro 4 menciona a dificuldade de alguns enfermeiros e profissionais de saúde na identificação de UP. Dois artigos ${ }^{(19,21)}$ retrataram o problemas para classificar estas lesões, especialmente as de grau I, visto Que não há descontinuidade na pele do paciente e algumas vezes esta lesão inicial não é percebida.

Além das limitações referentes ao estagiamento das UP pelos diversos profissionais da saúde, outra preocupação é a incidência das lesões relacionada ao despreparo dos profissionais na execução de cuidados para a prevenção de UP e Que comprovadamente não são assim caracterizados. Esse é o caso, por exemplo, da massagem, citada em um dos estudos da revisão, segundo a Qual é uma prática Que previne a desidratação, mas Que não tem indicação na prevenção de UP(22).

\section{CONSIDERAÇÕES FINAIS}

Para a Qualidade do cuidado de enfermagem é necessário uma formação de Qualidade voltada aos acadêmicos de enfermagem de 
modo Que estes se apropriem de conhecimentos científicos pertinentes a essa temática e se conscientizem da sua importância para uma assistência de enfermagem de Qualidade. Outro aspecto é a realização de educação em serviço e discussões acerca da temática de UP, para Que os profissionais, sejam capacitados e minimizem as lacunas na atuação nos diversos âmbitos de avaliação, prevenção e tratamento de UP.

Ressalta-se a importância do desenvolvimento de estudos clínicos direcionados principalmente à prevenção de UP para o fortalecimento das Práticas Baseadas em Evidências no campo de atuação da enfermagem, e consequentemente avanços científicos no conhecimento clínico construído pela enfermagem em nosso país.

Reconhecer as limitações envolvidas nesses aspectos e buscar superá-las proporcionará avanços e impulsionará a ampliação de estudos com fortes evidências clínicas Que subsidiam a prática profissional da enfermagem.

\section{REFERÊNCIAS}

1. Lobosco AAF.O enfermeiro atuando na prevenção das úlceras de Pressão. Enfermeria Global [serial online] 2008 Jun [cited 2009 April 20]:(1 3). Disponível em: http://www.um.es/eglobal/

2. European Pressure Ulcer Advisory Panel; 2007. [cited 2009 mai 5]. Available from: http://www.epuap.org/

3. Blanes I, Duarte IS, Calil IA, Ferreira LM. Avaliação clínica e epidemiológica das úlceras por pressão em pacientes internados no hospital São Paulo. Rev Assoc Med Bras 2004; 50(2): 1 82- 7.

4. Rogenski NMB, Santos VLCG. Estudo sobre a incidência de úlcera por pressão em um hospital universitário. Rev Latinoam Enfermagem 2005; 13(4): 474-80.

5. Galvão CM, Sawada NO, Mendes AC. A busca das melhores evidências. Rev Esc Enferm USP 2003; 37(4): 43-50.

6. Roman AR, Friedlander MR. Revisão integrativa de pesquisa aplicada à enfermagem. Cogitare Enferm 1998; 3(2): 109-12.

7. Ganong LH. Integrative Reviews of nursing research. Res Nur Health 1987; 10: 1-11.

8. Cruz DALM, Pimenta CAM. Prática baseada em evidências, aplicada ao raciocínio diagnóstico. Rev Latino-am Enfermagem 2005; 13(3).

9. Weng $\mathrm{MH}$. The effect of protective treatment in reducing pressure ulcers for non-invasive ventilation patients. Intensive and Critical Care Nursing 2008; 24(5): 295-9.

10. Feuchtinger J, Bic R, Dassen T, Halfens R. A 4-cm thermoactive viscoelastic foam pad on the operating room table to prevent pressure ulcer during cardiac surgery. I Clinical Nurs 2006; 15(2): 162-7.

11. Sheerin F, Frein R. The occipital and sacral pressure experienced by healthy volunteers under spinal immobilization: a trial of three surfaces. I Emerg Nurs 2007; 33(5): 447-50.

12. Jolley DI, Wright R, Macgowan S, Hickey MB, Campbell BA, Sinclair RD, et. al. Preventing pressure ulcers with the australian medical sheepskin: an open-label randomised controlled trial.Medical. I Australia 2004; I 80(7): 324-7.
13. Mistiaen P, Achterberg W, Ament A, Halfens R, Huizinga I, Montgomery K, et.al. Cost- effectiveness of Australian Medical sheepskin for the prevention of pressure ulcers in somatic nursing home patients: study protocol for a prospective multi-centre randomised controlled trial. BMC Health Serv Res 2008; 8(4).

14. Parnell LK, Ciufi B, Gokoo CF. Preliminary Use of hidrogel containing enzymes in the treatment of stage II and stage III pressure ulcers. Ostomy Wound Manag 2005; 5 I (8): 50-60.

15. Hodgkinson B, Rhonda N. Effectiveness of topical skin care provided in aged care facilities. Int I Evi Based Health Care 2005; 3: 65-101.

16. Fernandez R, Griffiths R, Ussia C. Effectiveness of solutions, techniques and pressure in wound cleansing. JBI Reports 2004; 2: $231-70$.

17. Defoor T, Grypdonk MFH. Pressure ulcers: validation of two risk assessment scales. I Clinical Nurs 2005; 14(3): 373-82.

18. Vanderwee K, Grypdonck M, Defloor T. Non - blanchable erythema as an indicator for the need for pressure ulcer prevention: a randomized - controlled trial. I Clinical Nurs 2007; 16(2): 325-35.

19. Nixon I, Thorpe H, Barrow H, Phillips A, Nelson E, Mason S, Cullum N. Reliability of pressure ulcer classification and diagnosis. J Advan Nurs 2005; 50(5): 613-23.

20. Wedge C, Gosney M. Pressure - relieving equipament: promoting its correct use amongst nurses via differing modes of educational delivery. I Clinical Nurs 2005, 14(4): 473-8.

21. Stausberg I, Lehmann N, Kroeger K. Reliability and validity of pressure ulcer diagnosis and granding: na image - based survey. Int I Nurs Studies 2007; 44(8): 1316-23.

22. Peeters I, Hulsenboom M, Berger M, Heh L, Halfens R. Massage to prevent pressure ulcers: knowledge, beliefs and practice. A cross-sectional study among nurses in the Netherlands in 1991 and 2003. I Clinical Nurs 2007; 15(4): 428-35. 


\section{APÊNDICE}

\begin{tabular}{|c|c|c|c|c|}
\hline ESTUDO & TIPO/AMOSTRA & $\begin{array}{c}\text { INTERVENÇÃO } \\
\text { ESTUDADA }\end{array}$ & RESULTADOS & CONCLUSÕES \\
\hline Weng ${ }^{91}$ & $\begin{array}{l}\text { Caso-controle, } 90 \\
\text { pacientes de unidades } \\
\text { de terapia intensiva. }\end{array}$ & $\begin{array}{l}\text { Comparar a eficácia dos curativos } \\
\text { Tegasorb e o Tegaderm* (grupos } \\
\text { experimentais) com o grupo controle } \\
\text { (sem curativo) na prevenção de UP em } \\
\text { região facial em pacientes sob } \\
\text { ventilação mecânica não invasiva. }\end{array}$ & $\begin{array}{l}\text { A proporção de pessoas } \\
\text { desenvolvendo úlcera por pressão } \\
\text { foi significativamente diferente nos } \\
\text { três grupos estudados. O tempo } \\
\text { para o desenvolvimento de úlcera } \\
\text { por pressão demonstrou ser menor } \\
\text { no grupo controle. }\end{array}$ & $\begin{array}{l}\text { O resultado revelou Que o grupo } \\
\text { Tegasorb e o Tegaderm podem } \\
\text { ser utilizados em face para } \\
\text { prevenir úlcera de pressão em } \\
\text { pacientes sob ventilação não } \\
\text { invasiva. }\end{array}$ \\
\hline Feuchtinger $^{(I 0)}$ & $\begin{array}{l}\text { Caso - controle; } \\
\text { I75 pacientes. }\end{array}$ & $\begin{array}{l}\text { Comparar uma espuma viscoelástica } \\
\text { termo ativa de } 4 \text { cm combinada a um } \\
\text { colchão de aquecimento disposto na } \\
\text { mesa da sala de operação, com uma } \\
\text { mesa padrão (colchão sem mecanismos } \\
\text { para redução de UP). }\end{array}$ & $\begin{array}{l}\text { Os resultados demostraram Que os } \\
\text { pacientes Que utilizaram a } \\
\text { cobertura viscoelástica ativa de } 4 \\
\text { cm combinada ao colchão de } \\
\text { aquecimento sofreram incidência } \\
\text { maior de UP (I7.6\%) Quando } \\
\text { comparados ao grupo Que utilizou } \\
\text { a mesa padrão }(11,1 \%) \text {. }\end{array}$ & $\begin{array}{l}\text { A combinação da espuma } \\
\text { viscoelástica ativa de } 4 \mathrm{~cm} \text { e } \\
\text { uma fonte de calor não pode ser } \\
\text { recomendada para prevenção de } \\
\text { UP em mesas de salas } \\
\text { operatórias. }\end{array}$ \\
\hline $\begin{array}{l}\text { Sheerin; } \\
\text { Frein }^{(I I)}\end{array}$ & $\begin{array}{l}\text { Ensaio clínico não } \\
\text { randomizado. Estudo } \\
\text { piloto envolvendo dois } \\
\text { voluntários. }\end{array}$ & $\begin{array}{l}\text { Verificar a pressão exercida em } \\
\text { pacientes Que sofreram lesão da } \\
\text { medula espinhal em } 3 \text { diferentes tipos } \\
\text { de superfície: tábua de imobilização } \\
\text { espinhal tradicional, tábua recoberta } \\
\text { com tecido inflável e colchão a vácuo } \\
\text { através de um sensor para incidência } \\
\text { de UP nas regióes occipital e sacra. }\end{array}$ & $\begin{array}{l}\text { As pressóes mensuradas na região } \\
\text { occipital foram maiores Quando } \\
\text { utilizada a tábua de imobilização } \\
\text { cervical tradicional. Na região } \\
\text { occipital as pressões mais baixas } \\
\text { foram registradas Quando o colchão } \\
\text { a vácuo foi utilizado. As pressões } \\
\text { mensuradas na região sacra foram } \\
\text { também significativamente maiores } \\
\text { Quando utilizado a tábua de } \\
\text { imobilização espinhal tradicional. }\end{array}$ & $\begin{array}{l}\text { Esse foi um estudo piloto para } \\
\text { futuras pesquisas. Se esses } \\
\text { achados forem confirmados } \\
\text { haverá implicaçôes no } \\
\text { gerenciamento do cuidado a } \\
\text { pacientes com lesão de medula } \\
\text { espinhal no primeiro estágio do } \\
\text { cuidado de enfermagem ao } \\
\text { adentrarem nas instituiçôes } \\
\text { hospitalares. }\end{array}$ \\
\hline $\begin{array}{l}\text { Jolleyet } \\
\text { al, }{ }^{(I 2)}\end{array}$ & $\begin{array}{l}\text { Ensaio clínico } \\
\text { controlado } \\
\text { randomizado; } 44 I \\
\text { pacientes. }\end{array}$ & $\begin{array}{l}\text { Pacientes foram randomizados para } \\
\text { receber um colchão com cobertura } \\
\text { sheepskin** durante sua permanência } \\
\text { no hospital e tratamento usual para } \\
\text { prevenção de UP. }\end{array}$ & $\begin{array}{l}58 \text { pacientes desenvolveram UP: } \\
2 \text { I no grupo sheepskin e } 37 \text { no } \\
\text { grupo controle. Pacientes no grupo } \\
\text { sheepskin desenvolveram novas UP } \\
\text { com uma taxa menor Que a metade } \\
\text { Quando comparada ao grupo } \\
\text { controle. }\end{array}$ & $\begin{array}{l}\text { O sheepskin é efetivo na redução } \\
\text { de incidências de UP em } \\
\text { pacientes de hospitais gerais } \\
\text { com baixo a moderado risco } \\
\text { para o desenvolvimento de UP. }\end{array}$ \\
\hline $\begin{array}{l}\text { Mistiaen et. } \\
a^{(13)}\end{array}$ & $\begin{array}{l}\text { Ensaio clínico } \\
\text { randomizado } \\
\text { controlado; amostra } \\
\text { prevista de } 750 \\
\text { pacientes }\end{array}$ & $\begin{array}{l}\text { A valiar os custos e os efeitos do } \\
\text { sheepskin Australiano combinado com } \\
\text { o cuidado tradicional na prevenção de } \\
\text { UP sacrais em pacientes de cuidado } \\
\text { domiciliar (grupo experimental) X } \\
\text { cuidado tradicional somente (grupo } \\
\text { controle). }\end{array}$ & $\begin{array}{l}\text { Uma discussão pode ser realizada } \\
\text { entre as variáveis relacionadas a } \\
\text { efetividade (incidência e } \\
\text { prevalência de UP) e variáveis } \\
\text { relacionadas ao custo (compra e } \\
\text { lavagem dos Sheepskin e } \\
\text { investimento de tempo e custo de } \\
\text { materiais para o tratamento de UP). }\end{array}$ & $\begin{array}{l}\text { O processo de inclusão de } \\
\text { pacientes iniciou em Março de } \\
2007 \text { e está previsto para } \\
\text { continuar até o final de } 2008 . \\
\text { Os primeiros resultados são } \\
\text { esperados para metade do ano } \\
\text { de } 2009 \text {. }\end{array}$ \\
\hline
\end{tabular}

* Tegasorb e Tegaderm são coberturas oclusivas Que criam uma barreira física sob a pele prevenindo o surgimento de lesões cutâneas.

** O sheepskin é uma superficie de couro Que contem aproximadamente $25 \mathrm{~mm}$ de lã natural. É utilizado como uma cobertura parcial do colchão e, é especificamente indicado para reduzir pressão, minimizar o cisalhamento e a friç̧ão bem como absorver a unidade:

\section{Quadro 1. Caracterização dos estudos, amostra, intervenção, resultados e conclusões relacionados a materiais utilizados na} prevenção de UP. 


\begin{tabular}{|c|c|c|c|c|}
\hline ESTUDO & TIPO/AMOSTRA & $\begin{array}{c}\text { INTERVENÇÃO } \\
\text { ESTUDADA }\end{array}$ & RESULTADOS & CONCLUSÕES \\
\hline $\begin{array}{l}\text { Parnell; } \\
\text { Ciufi; } \\
\text { Gokoo }^{(4)}\end{array}$ & $\begin{array}{l}\text { Um estudo prospectivo preliminar } \\
\text { de } 12 \text { semanas; } \\
\text { IO pacientes de cuidado } \\
\text { domiciliar com UP }\end{array}$ & $\begin{array}{l}\text { Avaliar o efeito da cobertura } \\
\text { contendo hidrogel com uma } \\
\text { combinação enzimas em } \\
\text { pacientes Que falharam e não } \\
\text { responderam a tratamentos } \\
\text { anteriormente utilizados na } \\
\text { cura de UP. }\end{array}$ & $\begin{array}{l}\text { Sete pacientes (três com estágio II } \\
\text { e com estágio III) completaram o } \\
\text { estudo.A cura foi baseada no } \\
\text { critério de fechamento da úlcera } \\
\text { pela re-epitelização como } \\
\text { determinado pela mensuração da } \\
\text { área e avaliação clínica. Todas as } \\
\text { três úlceras de estágio II e duas de } \\
\text { estágio III foram completamente } \\
\text { curadas. } 4 \text { úlceras de estágio III } \\
\text { foram categorizadas como em } \\
\text { processo de cura. }\end{array}$ & $\begin{array}{l}\text { Estudos adicionais para definir } \\
\text { possíveis contribuições de } \\
\text { enzimas na cura de feridas são } \\
\text { recomendados visto os dados } \\
\text { encontrados nesse estudo. }\end{array}$ \\
\hline $\begin{array}{l}\text { Hodgkinson; } \\
\text { Rhonda(15) }^{\text {Hond }}\end{array}$ & Revisão sistemática de literatura. & $\begin{array}{l}\text { O objetivo da revisão foi } \\
\text { determinar a melhor } \\
\text { evidência disponível para a } \\
\text { efetividade e segurança de } \\
\text { aplicabilidade de agentes } \\
\text { tópicos na pele de idosos } \\
\text { com enfoQue a prevenção de } \\
\text { UP. }\end{array}$ & $\begin{array}{l}\text { As evidências foram variáveis } \\
\text { dependendo das condiçôes da } \\
\text { pele. }\end{array}$ & $\begin{array}{l}\text { Ainda há lacunas acerca dos } \\
\text { estudos envolvendo agentes } \\
\text { tópicos. Devido a falta de } \\
\text { evidência nos estudos, nenhuma } \\
\text { recomendação sobre segurança } \\
\text { sobre intervenções puderam ser } \\
\text { incluídas nesta revisão. }\end{array}$ \\
\hline $\begin{array}{l}\text { Fernadez; } \\
\text { Griffiths; } \\
\text { Ussia(16) }^{(16}\end{array}$ & $\begin{array}{l}\text { Revisão sistemática da literatura } \\
\text { utilizando ensaios clínicos } \\
\text { controlados e ramdomizados } \\
\text { identificados pela Cochrane } \\
\text { Central Register of Controlled } \\
\text { Trial (CENTRAL). }\end{array}$ & $\begin{array}{l}\text { Avaliar a efetividade de } \\
\text { diferentes soluçôes, } \\
\text { procedimentos e técnicas } \\
\text { utilizados na limpeza da } \\
\text { ferida para prevenir a } \\
\text { infecção e promover a sua } \\
\text { cura. }\end{array}$ & $\begin{array}{l}\text { A evidência indica Que não há } \\
\text { diferença entre as taxas de infecção } \\
\text { e cura em feridas agudas e crônicas } \\
\text { Quando limpas tanto com água ou } \\
\text { com solução salina fisiológica. Não } \\
\text { há estudos comparando técnicas } \\
\text { comuns de limpeza de feridas. }\end{array}$ & $\begin{array}{l}\text { Há evidências disponíveis para } \\
\text { uso de soluções e técnicas } \\
\text { destinadas à limpeza da ferida. }\end{array}$ \\
\hline
\end{tabular}

Quadro 2. Caracterização dos estudos, amostra, intervenção, resultados e conclusões relacionados a diferentes tratamentos utilizados em UP.

\begin{tabular}{|c|c|c|c|c|}
\hline ESTUDO & TIPO/AMOSTRA & $\begin{array}{c}\text { INTERVENÇÄO } \\
\text { ESTUDADA }\end{array}$ & RESULTADOS & CONCLUSÕES \\
\hline $\begin{array}{l}\text { Defoor; } \\
\text { Grypdonk }\end{array}$ & Ensaio clínico; 1772 pacientes & $\begin{array}{l}\text { Comparar o valor preditivo e } \\
\text { a efetividade das } \\
\text { mensurações preventivas de } \\
\text { duas escalas de avaliação de } \\
\text { risco para desenvolvimento } \\
\text { de úlcera por pressão } \\
\text { (Braden e Norton) aliadas ao } \\
\text { julgamento clínico realizado } \\
\text { pelas enfermeiras. }\end{array}$ & $\begin{array}{l}\text { Analisando os itens das escalas em } \\
\text { uma análise de regressão logística } \\
\text { obtiveram-se diferentes resultados. } \\
\text { Nenhum dos itens da escala de } \\
\text { Braden predisse o aparecimento de } \\
\text { lesão por úlcera de pressão e na } \\
\text { escala de Norton somente o item } \\
\text { 'atividade'. }\end{array}$ & $\begin{array}{l}\text { Se as enfermeiras agirem de } \\
\text { acordo com as escalas de } \\
\text { avaliação de risco estudadas, } \\
80 \% \text { dos pacientes deverão } \\
\text { receber desnecessariamente } \\
\text { medidas de prevenção. O uso de } \\
\text { medidas preventivas diminui o } \\
\text { valor preditivo das escalas. }\end{array}$ \\
\hline $\begin{array}{l}\text { Vanderwee; } \\
\text { Grypdonck; } \\
\text { Defloor }^{(18)}\end{array}$ & $\begin{array}{l}\text { Ensaio clínico randomizado; } \\
\text { multicêntrico, em } 14 \text { unidades } \\
\text { dispostas em } 6 \text { hospitais da } \\
\text { Bélgica. }\end{array}$ & $\begin{array}{l}\text { Avaliar se as medidas de } \\
\text { prevenção de UP Quando } \\
\text { postergadas atéo } \\
\text { aparecimento do eritema não } \\
\text { branqueável aumentam a } \\
\text { incidência da UP Quando } \\
\text { comparadas com os métodos } \\
\text { de avaliação de escalas de } \\
\text { risco. }\end{array}$ & $\begin{array}{l}\text { No grupo experimental, I6\% dos } \\
\text { pacientes receberam medidas } \\
\text { preventivas e no grupo controle } \\
32 \% \text {. A incidência de UP não foi } \\
\text { significativa entre os dois grupos. }\end{array}$ & $\begin{array}{l}\text { Poucos pacientes necessitam de } \\
\text { medidas preventivas Quando a } \\
\text { prevenção é postergada até o } \\
\text { apare cimento do eritema não } \\
\text { branqueável e esses pacientes } \\
\text { não desenvolvem mais UP do } \\
\text { Que pacientes Que receberam } \\
\text { prevenção baseados em métodos } \\
\text { de avaliação de risco } \\
\text { padronizados. }\end{array}$ \\
\hline
\end{tabular}

Quadro 3. Caracterização dos estudos, amostra, intervenção, resultados, conclusões Que utilizam as escalas de avaliação de risco para o desenvolvimento de UP. 


\begin{tabular}{|c|c|c|c|c|}
\hline ESTUDO & TIPO/AMOSTRA & $\begin{array}{c}\text { INTERVENÇÃO } \\
\text { ESTUDADA }\end{array}$ & RESULTADOS & CONCLUSÕES \\
\hline $\begin{array}{l}\text { Nixon et } \\
\text { al }^{(19)}\end{array}$ & $\begin{array}{l}\text { Ensaio clínico; } \\
378 \text { avaliações pareadas }\end{array}$ & $\begin{array}{l}\text { Pacientes tiveram a pele } \\
\text { avaliada em sete sítios por } \\
\text { diferentes profissionais } \\
\text { enfermeiras. O objetivo foi } \\
\text { averiguar se apesar do } \\
\text { treinamento recebido por } \\
\text { todas as enfermeiras, a } \\
\text { avaliação das UP foi realizada } \\
\text { de maneira similar. }\end{array}$ & $\begin{array}{l}\text { Houve I00\% de concordância } \\
\text { entre os diagnósticos de úlcera por } \\
\text { pressão entre as enfermeiras } \\
\text { pesquisadoras e as enfermeiras } \\
\text { gerentes, e o método estatístico } \\
\text { Kappa* indicou uma concordância } \\
\text { "muito boa. Houve variação nos } \\
\text { sítios de avaliação da pele, entre } \\
93.6 \% \text { e IO0\% com a estatística } \\
\text { Kappa indicando concordância } \\
\text { boa' ou 'muito boa'. }\end{array}$ & $\begin{array}{l}\text { Apesar da estatística Kappa } \\
\text { apontar concordâncias } \\
\text { significativas ainda há uma alta } \\
\text { prevalência de discordância nos } \\
\text { diagnósticos de úlcera por } \\
\text { pressão principalmente da } \\
\text { identificação do grau I (eritema } \\
\text { não branQueável) e grau } 2 \text { de } \\
\text { úlcera por pressão. }\end{array}$ \\
\hline $\begin{array}{l}\text { Wedge; } \\
\text { Gosney }^{(20)}\end{array}$ & $\begin{array}{l}\text { Ensaio clínico randomizado pré e } \\
\text { pós teste; } \\
12 \text { alas concordaram em participar } \\
\text { do estudo e } 245 \text { leitos foram } \\
\text { examinados }\end{array}$ & $\begin{array}{l}\text { Comparar a eficácia de um } \\
\text { treinamento escrito a um } \\
\text { treinamento escrito e verbal } \\
\text { sobre as práticas de preparo } \\
\text { do leito com a utilização de } \\
\text { tecnologias para prevenção } \\
\text { de UP. }\end{array}$ & $\begin{array}{l}\text { Antes do treinamento } 46 \% \text { dos } \\
\text { leitos tinham lençóis dispostos } \\
\text { corretamente nos colchões. Após o } \\
\text { treinamento essa porcentagem } \\
\text { aumentou para } 87,8 \% \text {. Não houve } \\
\text { diferença significativa no número } \\
\text { de leitos preparados corretamente } \\
\text { nos dois diferentes grupos de } \\
\text { educação. }\end{array}$ & $\begin{array}{l}\text { Claramente a instrução inscrita } \\
\text { melhorou a prática, mas a } \\
\text { educação verbal não teve } \\
\text { nenhum benefício adicional. }\end{array}$ \\
\hline $\begin{array}{l}\text { Stausberget } \\
\text { al }^{(2)}\end{array}$ & $\begin{array}{l}\text { Cross sectional survey; } \\
\text { dois médicos e Quatro enfermeiras }\end{array}$ & $\begin{array}{l}\text { Avaliar a concordância entre } \\
\text { IOO imagens de úlceras por } \\
\text { pressão e outras lesões na } \\
\text { pele bem como seus vários } \\
\text { graus de estagiamento. Os } \\
\text { locais avaliados foram } \\
\text { pés, calcanhares, nádegas e } \\
\text { Quadris }\end{array}$ & $\begin{array}{l}\text { A porcentagem de concordância } \\
\text { entre esses profissionais foi maior } \\
\text { para o diagnóstico de UP nas } \\
\text { regióes de pés e calcanhares e } \\
\text { menor na avaliação do } \\
\text { estadiamento de UP nas regiões de } \\
\text { nádegas e Quadris. Nenhuma } \\
\text { diferença estatística significante } \\
\text { pode ser encontrada entre as } \\
\text { enfermeiras e os médicos Que } \\
\text { avaliaram as lesões. }\end{array}$ & $\begin{array}{l}\text { A avaliação do estagiamento de } \\
\text { UP é mais fácil nas regiões de } \\
\text { pés e calcanhares do que em } \\
\text { regióes de nádegas e Quadris. É } \\
\text { mais difícil fazer o diagnóstico } \\
\text { de UP do que graduá-las em seu } \\
\text { estagiamento. }\end{array}$ \\
\hline $\begin{array}{l}\text { Peeters et al } \\
\text { (22) }\end{array}$ & $\begin{array}{l}\text { Survey com529 enfermeiras } \\
\text { preencheram Questionários } \\
\text { formulados com enfoque sobre o } \\
\text { seu conhecimento, crenças e } \\
\text { métodos de prevenção em UP. }\end{array}$ & $\begin{array}{l}\text { Comparar o atual } \\
\text { conhecimento relacionado às } \\
\text { crenças e práticas envolvendo } \\
\text { a técnica de massagem por } \\
\text { meio da comparação com o } \\
\text { primeiro guideline } \\
\text { publicado no país em } 1991 \text { e } \\
\text { a sua nova versão publicada } \\
\text { em } 2002 \text {. }\end{array}$ & $\begin{array}{l}\text { Os resultados mostram uma } \\
\text { mudança significativa no } \\
\text { conhecimento, crenças e práticas } \\
\text { envolvendo a técnica de massagem. } \\
\text { EnQuanto em } 199187,4 \% \text { das } \\
\text { enfermeiras diziam Que 'sempre'ou } \\
\text { algumas vezes' usavam a } \\
\text { massagem para prevenção de UP, } \\
\text { em } 2003 \text { essa percentagem } \\
\text { reduziu para } 48,9 \text {. }\end{array}$ & $\begin{array}{l}\text { As diferenças nas respostas entre } \\
1991 \text { e } 2003 \text { são significantes e } \\
\text { sugerem que as enfermeiras } \\
\text { estavam melhores informadas em } \\
2003 .\end{array}$ \\
\hline
\end{tabular}

* O método estatístico Kappa pode verificar se a concordância analisada entre os grupos em comparação excede o padrão como referência para um dos grupos. A estatística deste método é influenciada pela prevalência nas categorias em análise.

Quadro 4. Caracterização dos estudos (amostra, intervenção, resultados, conclusões) Que tratam das comparações entre a concordância de profissionais de saúde acerca das práticas clínicas relacionadas às UP. 